\title{
SYSTOLE ON LOCALLY SYMMETRIC SPACES
}

\author{
INKANG KIM
}

\begin{abstract}
Here we survey on the growth of systoles of arithmetic locally symmetric spaces under the congruence covering and give simple proofs for the best possible constants of Gromov for several important classes of symmetric spaces.
\end{abstract}

\section{Contents}

Introduction 1

1. Real rank one case 3

1.1. Real and Quaternionic hyperbolic space 3

1.2. Complex hyperbolic space 5

2. Arithmetic lattice in $S L(n, \mathbb{R}) \quad 7$

3. Uniform lower bound for systoles of nonuniform arithmetic manifolds in complex hyperbolic space $\quad 8$

$\begin{array}{ll}\text { References } & 10\end{array}$

\section{INTRODUCTION}

A systole $\operatorname{sys}_{1}(M)$ of a Riemannian manifold $M$ is the smallest length of closed geodesics in $M$. It captures certain aspect of geometry of $M$. For example, Margulis conjectured that there exists a uniform lower bound of systoles for arithmetic locally symmetric manifolds of finite volume. This conjecture has been supported by several cases. For example, the systole grows under the covering map, which was demonstrated, not long ago by Buser and Sarnak in [1], where they constructed examples of congruence coverings of an arithmetic hyperbolic surface whose systole grows logarithmically with respect to the area:

$$
\operatorname{sys}_{1}(S) \geq \frac{4}{3} \log (\operatorname{area}(S))-c,
$$

where $c$ is a constant independent of $S$. In 1996, Gromov [5, Sec. 3.C.6] showed that for any regular congruence covering $M_{I}$ of a compact arithmetic locally

\footnotetext{
${ }^{1} 2000$ Mathematics Subject Classification. 53C43, 53C21, 53C25

${ }^{2}$ Key words and phrases. Systole, arithmetic lattice, locally symmetric space.

${ }^{3}$ Research by the author is partially supported by Grant NRF-2017R1A2A2A05001002.
} 
symmetric space $M$, there exists a constant $C>0$ so that

$$
\operatorname{sys}_{1}\left(M_{I}\right) \geq C \log \left(\operatorname{vol}\left(M_{I}\right)\right)-d,
$$

where $d$ is independent of $M_{I}$. Nonetheless, an explicit value for the constant $C$ is unkown. The purpose of this short paper is to provide the best possible constant $C$ for some locally symmetric spaces. We call $C$ a Gromov constant.

Our method is extremely simple in the sense that the techniques only use length-trace inequality, norm of a prime ideal in algebraic integers of a totally real number field, and the size of a group over finite field. Especially we will use the following table throughout the paper.

TABLE 1. Size of classical semisimple groups over $\mathbb{F}_{q}$

\begin{tabular}{cc}
\hline \hline type of $G$ & cardinality of $G\left(\mathbb{F}_{q}\right)$ \\
\hline${ }^{1} A_{r}(r \geq 1)$ & $q^{r(r+1) / 2} \Pi_{j=1}^{r}\left(q^{j+1}-1\right)$ \\
${ }^{2} A_{r}(r \geq 2)$ & $q^{r(r+1) / 2} \Pi_{j=1}^{r}\left(q^{j+1}-(-1)^{j=1}\right)$ \\
$B_{r}$ or $C_{r}(r \geq 2)$ & $q^{r^{2}} \Pi_{j=1}^{r}\left(q^{2 j}-1\right)$ \\
${ }^{1} D_{r}(r \geq 4)$ & $q^{r(r-1)}\left(q^{r}-1\right) \Pi_{j=1}^{r-1}\left(q^{2 j}-1\right)$ \\
${ }^{2} D_{r}(r \geq 4)$ & $q^{r(r-1)}\left(q^{r}+1\right) \Pi_{j=1}^{r-1}\left(q^{2 j}-1\right)$ \\
\hline
\end{tabular}

For a given arithmetic lattice $\Gamma=\mathbf{G}\left(\mathcal{O}_{F}\right)$ defined over totally real number field $F$, we will consider the congruence coverings corresponding to prime ideals $I \subset \mathcal{O}_{F}$, i.e.

$$
\Gamma(I)=\Gamma \cap \operatorname{ker}\left(\mathbf{G}\left(\mathcal{O}_{F}\right) \rightarrow \mathbf{G}\left(\mathcal{O}_{F} / I\right)\right)
$$

via suitable embedding of $\mathbf{G}$ into some linear group. Denote the corresponding cover of $M$ by $M_{I}$. We will estimate $C$ in the inequality

$$
\operatorname{sys}_{1}\left(M_{I}\right) \geq C \log \left(\operatorname{vol}\left(M_{I}\right)\right)-c,
$$

where $c$ is independent of $I \subset \mathcal{O}_{F}$.

Theorem 0.1. $C$ depends on the type of $G$.

(1) For arithmetic lattices in $S O(1, n), C=\frac{4}{n(n+1)}$.

(2) For arithmetic lattices in $S U(n, 1)$ of the first type, $C=\frac{4}{n(n+2)}$, of the other type, $C=\frac{2}{n(n+2)}$.

(3) For arithmetic lattices in $S L(n+1, \mathbb{R}), C=\frac{\sqrt{2}}{n(n+2)}$.

In the last section, we deal with the uniform lower bound for systoles of nonuniform arithmetic complex hyperbolic lattices using complex Salem number. 


\section{ReAl RANK ONE CASE}

1.1. Real and Quaternionic hyperbolic space. For arithmetic lattices of the first type in real hyperbolic case, Murillo [9] proved that

$$
\operatorname{sys}_{1}\left(T_{I}\right) \geq \frac{8}{n(n+1)} \log \left(\operatorname{vol}\left(T_{I}\right)\right)-d .
$$

For quaternionic hyperbolic lattice, Emery-Kim-Murillo [2] proved

$$
\operatorname{sys}_{1}\left(M_{I}\right) \geq \frac{4}{(n+1)(2 n+3)} \log \left(\operatorname{vol}\left(M_{I}\right)\right)-d .
$$

We give a systole growth inequality for real hyperbolic cases which have not been covered yet. Let $F$ be a totally real number field and $\mathcal{O}_{F}$ the ring of integers of $F$. Let $a_{1}, \cdots, a_{n} \in \mathcal{O}_{F}$ such that $a_{i}>0$ for all $i$, but $\sigma\left(a_{j}\right)<0$ for any Galois embedding $\sigma \neq i d$. Then $G=S O\left(x_{0}^{2}-a_{1} x_{1}^{2}-\cdots-a_{n} x_{n}^{2}, \mathbb{R}\right)=S O(1, n)$, and $G\left(\mathcal{O}_{F}\right)$ is an arithmetic lattice of $G$. Even though this construction is exhaustive when $n$ is even, for $n$ odd, we need another construction using quaternion algebra.

Let $\mathbb{H}_{F}^{a, b}$ be a quaternion algebra over $F$. Set

$$
\tau_{r}\left(x_{0}+x_{1} i+x_{2} j+x_{3} k\right)=x_{0}+x_{1} i-x_{2} j+x_{3} k .
$$

For $A \in G L\left(m, \mathbb{H}_{F}^{a, b}\right)$ with $\tau_{r}\left(A^{T}\right)=A$, let

$$
S U\left(A, \tau_{r}, \mathbb{H}_{F}^{a, b}\right)=\left\{g \in S L\left(m, \mathbb{H}_{F}^{a, b}\right) \mid \tau_{r}\left(g^{T}\right) A g=A\right\} .
$$

Let $a$ and $b$ are nonzero real numbers such that either $a$ or $b$ is positive and $x$ is an invertible element of $\mathbb{H}_{\mathbb{R}}^{a, b}$ such that $x=p+q i+s k$. Define the norm

$$
N(x)=x \bar{x}=p^{2}-a q^{2}+a b s^{2},
$$

and

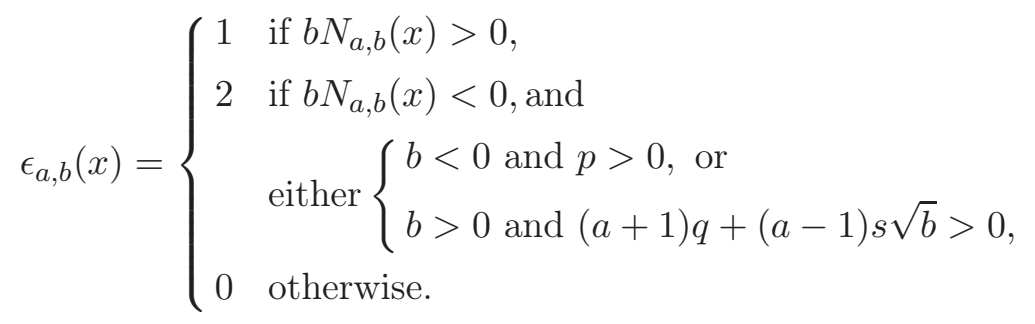

Let $a, b \in F^{\times}$such that for each Galois embedding $\sigma$ of $F$, either $\sigma(a)$ or $\sigma(b)$ is positive, and choose $a_{1}, \cdots, a_{m} \in \mathbb{H}_{F}^{a, b}$ such that

(1) $\tau_{r}\left(a_{l}\right)=a_{l}$

(2) $\sigma\left(a_{l}\right)$ is invertible for each $l$ and $\sigma$

(3) $\sum_{l=1}^{m} \epsilon_{a, b}\left(a_{l}\right)=1$, and

(4) $\sum_{l=1}^{m} \epsilon_{\sigma(a), \sigma(b)}\left(\sigma\left(a_{l}\right)\right) \in\{0,2 m\}$ for each $\sigma \neq i d$, Then $\mathbf{G}=S U\left(\operatorname{diag}\left(a_{1}, \cdots, a_{m}\right), \tau_{r}, \mathbb{H}_{F}^{a, b}\right)^{0}=S O(1,2 m-1)^{0}$ and $\Gamma=\mathbf{G}(\mathcal{O})$ is an arithmetic lattice of $\mathbf{G}$ where $\mathcal{O}$ is an order of $\mathbb{H}_{F}^{a, b}$. For details, see [8]. As a vector space over $F,\left(\mathbb{H}_{F}^{a, b}\right)^{m}$ is isomorphic to $F^{4 m}$, and hence one 
can identify $G L\left(m, \mathbb{H}_{F}^{a, b}\right)$ with $G L(4 m, F)$. Under this identification, $\mathbf{G}(\mathcal{O})$ sits inside $G L\left(4 m, \mathcal{O}_{F}\right)$.

A hyperbolic isometry in $S O(1, n)$ can be conjugate to a form

$$
\left(\begin{array}{ccc}
S O(n-1) & 0 & 0 \\
0 & \cosh r & \sinh r \\
0 & \sinh r & \cosh r
\end{array}\right)
$$

whose translation length $\ell_{A}$ is $2 r$. Let $\lambda_{1}, \cdots, \lambda_{n_{1}}$ be eigenvalues of $S O(n-1)$ part of $A$. Then

$$
|\operatorname{tr} A|=\left|\lambda_{1}+\cdots+\lambda_{n-1}+2 \cosh r\right| \leq(n-1)+2 e^{r},
$$

hence

$$
|\operatorname{tr} A| \leq(n-1)+2 e^{\frac{\ell_{A}}{2}} .
$$

We get

$$
\ell_{A} \geq 2 \log \frac{|\operatorname{tr} A|-(n-1)}{2} .
$$

For any $A=\left(a_{i j}\right) \in \Gamma(I)$ for a prime ideal $I \subset \mathcal{O}_{F}$, where

$$
\Gamma(I)=\Gamma \cap \operatorname{ker}\left(M\left(4 m, \mathcal{O}_{F}\right) \rightarrow M\left(4 m, \mathcal{O}_{F} / I\right)\right),
$$

$a_{i i}=1+c_{i}$ with $c_{i} \in I$, and since $G^{\sigma}$ is compact for any Galois embedding $\sigma \neq i d$ of $F$,

$$
\left|\operatorname{tr} A^{\sigma}\right|=\left|\sum \sigma\left(1+c_{i}\right)\right|=\left|4 m+\sum \sigma\left(c_{i}\right)\right| \leq 4 m .
$$

Hence $\left|\sum \sigma\left(c_{i}\right)\right| \leq 8 m$. This impies

$$
N(I) \leq N\left(\sum c_{i}\right)=\left|\sum c_{i}\right| \Pi_{\sigma \neq i d}\left|\sigma\left(\sum c_{i}\right)\right| \leq(8 m)^{f-1}\left|\sum c_{i}\right|,
$$

where $f$ is a degree of $F$ over $\mathbb{Q}$. Then

$$
|\operatorname{tr} A|=\left|4 m+\sum c_{i}\right| \geq\left|\sum c_{i}\right|-4 m \geq \frac{N(I)}{(8 m)^{f-1}}-4 m,
$$

which implies

$$
\ell_{A} \geq 2 \log \frac{|\operatorname{tr} A|-(2 m-1-1)}{2} \geq 2 \log N(I)-c .
$$

Then we get

$$
\operatorname{sys}_{1}\left(M_{I}\right) \geq 2 \log (\mathrm{N}(I))-c,
$$

where $c$ is independent of $I \subset \mathcal{O}_{K}$. But $\mathbf{G}=S O(1, n=2 m-1)$ is of type ${ }^{2} D_{m}$, hence accroding to the Table 1

$$
\left|\mathbf{G}\left(\mathcal{O}_{k} / I\right)\right| \leq \mathrm{N}(I)^{m(2 m-1)} .
$$

From $\operatorname{vol}\left(M_{I}\right)=\operatorname{vol}(M)\left[\Gamma: \Gamma_{I}\right]$ and

$$
\begin{gathered}
0 \rightarrow \Gamma(I) \rightarrow \Gamma=\mathbf{G}\left(\mathcal{O}_{F}\right) \rightarrow \mathbf{G}\left(\mathcal{O}_{F} / I\right) \rightarrow 0, \\
{\left[\Gamma: \Gamma_{I}\right] \leq\left|S O\left(1, n ; \mathcal{O}_{F} / I\right)\right| \leq N(I)^{m(2 m-1)},}
\end{gathered}
$$


for an arithmetic real hyperbolic manifold of dimension $n=2 m-1 \geq 7$

$$
\operatorname{sys}_{1}\left(M_{I}\right) \geq \frac{4}{n(n+1)} \log \left(\operatorname{vol}\left(M_{I}\right)\right)-d \text {. }
$$

Note that for real hyperbolic manifold of dimension $n=2 m \geq 4$, it is of type $B_{m}$, and

$$
\left|\mathbf{G}\left(\mathcal{O}_{k} / I\right)\right| \leq \mathrm{N}(I)^{m(2 m+1)}
$$

Hence

$$
\operatorname{sys}_{1}\left(M_{I}\right) \geq \frac{4}{n(n+1)} \log \left(\operatorname{vol}\left(M_{I}\right)\right)-d .
$$

In any case, the constant is half of the one given by Murillo.

\subsection{Complex hyperbolic space.}

1.2.1. Arithmetic lattices in $S U(n, 1)$. Let $\Gamma \subset S U(n, 1), n>1$ be an arithmetic lattice of the first type defined over a totally real number field $K$ with a totally complex quadratic extension field $l$ such that $\Gamma$ is commensurable to $S U(h)\left(\mathcal{O}_{l}\right)$ where $h$ is a non-degenerate admissible Hermitian form over $V=l^{n+1}$ of signature $(n, 1)$. By a proper embedding of $S U(h)$ in $G L_{N}$ for large $N$, we can assume that an element in $S U(h)\left(\mathcal{O}_{l}\right)$ is a matrix in $G L_{N}\left(\mathcal{O}_{K}\right)$. It suffices to take $N=2 n+2$ as we can see below.

Another way to describe an arithmetic lattice of the first type defined over $K$ is as follows. Let $h$ be a Hermitian form over $l$ with coefficient matrix $A=\left(a_{i j}\right)$, i.e.,

$$
h(z)=z^{*} A z
$$

with $a_{i j} \in l$. Then

$$
\mathbf{G}=U(h, l)=\left\{T \in G L(n+1, l) \mid T^{*} A T=A\right\} .
$$

Using the restriction of scalars $R_{l \mid K}(\mathbf{G}), \mathbf{G}(l)$ corresponds to $R_{l \mid K}(\mathbf{G})(K)$, and we can ensure that $U(h, l)$ sits inside $G L(2 n+2, K)$. Under this correspondence, $S U(h)\left(\mathcal{O}_{l}\right)$ sits inside $G L\left(2 n+2, \mathcal{O}_{K}\right)$. The form $h$ is admissible if it has signature $(n, 1)$, and for any non-identity embedding $\sigma: l \rightarrow \mathbb{R}$, the Hermitian form $h^{\sigma}$ has signature $n+1$.

An arithmetic lattice of the second type is constructed as follows. Let $L$ be a cyclic extension of $l$ and choose embeddings $\lambda_{j}: L \rightarrow \mathbb{C}$ compatible with $\tau_{i}: l \rightarrow \mathbb{C}$ extending $\sigma_{i}: K \rightarrow \mathbb{R}$. Identify $L \rightarrow \mathbb{C}$ via $\lambda_{1}$. Given a cyclic l-algebra $A$, identify $A \otimes_{l} L=M(n+1, L)$. For each $\lambda_{i} \neq \lambda_{1}$, one obtains a new algebra $A^{\lambda_{i}}$. Now take a unitary division algebra $(A, *)$ of degree $n+1$ over $l$, with a Hermitian element $h$ (i.e., $h^{*}=h$ ) of signature $(n, 1)$. Let $x^{\star h}=\mu_{h} \circ x^{*}=h x^{*} h$ and

$$
S U(h)=\left\{x \in\left(A \otimes_{l} \mathbb{C}\right)^{\times}: x x^{\star h}=1\right\}=S U(n, 1) .
$$

If $h$ is admissible, i.e. $S U\left(h^{\tau_{j}}\right)$ is compact for $j \neq 1$, then

$$
S U(h, \mathcal{O})=\left\{x \in \mathcal{O}^{\times}: x x^{\star h}=1\right\}
$$


is a cocompact lattice in $S U(n, 1)$ where $\mathcal{O}$ is an $\mathcal{O}_{l}$ order in $A$. This arithmetic lattice is called of the second type. Even in this case, under the embedding $A \rightarrow A \otimes_{l} L=M(n+1, L)$, an element in $S U(h, \mathcal{O})$ lands in $M\left(n+1, \mathcal{O}_{l}\right)$, and finally in $M\left(2 n+2, \mathcal{O}_{K}\right)$.

Finally we introduce the mixed type arithmetic lattices. Let $(A, *)$ be a unitary algebra over $l$ of degree $d$. The simple $l$-algebra $M(r, A)$ admits an involution of second kind given by $*$-transposition. If $L$ is a splitting field for $A$, then

$$
M(r, A) \otimes_{l} L=M\left(r, A \otimes_{l} L\right)=M(r, M(d, L))=M(r d=n+1, L) .
$$

If a Hermitian element $h \in M(r, A)$ has signature $(n, 1)$ in the splitting $M(r, A) \otimes_{l}$ $L, S U\left(h, A \otimes_{l} \mathbb{C}\right)=S U(n, 1)$ and if $S U\left(h^{\tau_{j}}, A \otimes_{l} \mathbb{C}\right)$ is compact for any $\tau_{j} \neq \tau_{1}$, then $S U(h, \mathcal{O})$ is an arithmetic lattice in $S U(n, 1)$ for any $\mathcal{O}_{l}$-order $\mathcal{O}$. Under the embedding $A \rightarrow A \otimes_{l} L=M(d, L)$, an element in $S U(h, \mathcal{O})$ lands in $M\left(n+1, \mathcal{O}_{l}\right)$, and finally in $M\left(2 n+2, \mathcal{O}_{K}\right)$. For arithmetic lattices in complex hyperbolic space, refer to [7].

1.2.2. Systole growth. If $A \in S U(n, 1)$ is hyperbolic, it is conjugate to a matrix of the form

$$
\left(\begin{array}{lll}
U & 0 & 0 \\
0 & \lambda & 0 \\
0 & 0 & \beta
\end{array}\right)
$$

where $U \in U(n-1), \lambda=\overline{\beta^{-1}}$ with $|\lambda|>1$ and the other eigenvalues $\lambda_{1}, \cdots, \lambda_{n-1}$ all lie on the unit circle. The translation length of $A$ is

$$
\ell_{A}=2 \log |\lambda| \text {. }
$$

Since

$$
\begin{gathered}
|\operatorname{tr} A|=\left|\lambda_{1}+\cdots+\lambda_{n-1}+\lambda+\beta\right| \leq(n-1)+2|\lambda|, \\
\ell_{A} \geq 2 \log \frac{|\operatorname{tr} A|-(n-1)}{2} .
\end{gathered}
$$

For any $A=\left(a_{i j}\right) \in \Gamma(I)$ for any prime ideal $I \subset \mathcal{O}_{K}$, where

$$
\Gamma(I)=\Gamma \cap \operatorname{ker}\left(M\left(2 n+2, \mathcal{O}_{K}\right) \rightarrow M\left(2 n+2, \mathcal{O}_{K} / I\right)\right),
$$

$a_{i i}=1+c_{i}$ with $c_{i} \in I$,

$$
\left|\operatorname{tr} A^{\sigma}\right|=\left|\sum \sigma\left(1+c_{i}\right)\right|=\left|2 n+2+\sum \sigma\left(c_{i}\right)\right| \leq 2 n+2 .
$$

Hence $\left|\sum \sigma\left(c_{i}\right)\right| \leq 4 n+4$. This impies

$$
N(I) \leq N\left(\sum c_{i}\right)=\left|\sum c_{i}\right| \Pi_{\sigma \neq i d}\left|\sigma\left(\sum c_{i}\right)\right| \leq(4 n+4)^{f-1}\left|\sum c_{i}\right|,
$$

where $f$ is a degree of $K$ over $\mathbb{Q}$. Then

$$
|\operatorname{tr} A|=\left|2 n+2+\sum c_{i}\right| \geq\left|\sum c_{i}\right|-2 n-2 \geq \frac{N(I)}{(4 n+4)^{f-1}}-2 n-2,
$$


hence

$$
\ell_{A} \geq 2 \log \frac{|\operatorname{tr} A|-(n-1)}{2} \geq 2 \log \left[\frac{N(I)}{2(4 n+4)^{f-1}}-\frac{3 n+1}{2}\right] \geq 2 \log N(I)-c .
$$

Hence we obtain

$$
\operatorname{sys}_{1}\left(M_{I}\right) \geq 2 \log (\mathrm{N}(I))-c,
$$

where $c$ is independent of $I \subset \mathcal{O}_{K} \cdot \mathbf{G}=S U(n, 1)$ is of type ${ }^{2} A_{n}$, hence

$$
\left|\mathbf{G}\left(\mathcal{O}_{k} / I\right)\right| \leq \mathrm{N}(I)^{n(n+2)} .
$$

Finally we obtain for an arithmetic complex hyperbolic manifold

$$
\operatorname{sys}_{1}\left(M_{I}\right) \geq \frac{2}{n(n+2)} \log \left(\operatorname{vol}\left(M_{I}\right)\right)-d \text {. }
$$

For an arithmetic lattice of the first type, more precise estimates in [2] goes through for this case, and one obtains

$$
\operatorname{sys}_{1}\left(M_{I}\right) \geq \frac{4}{n(n+2)} \log \left(\operatorname{vol}\left(M_{I}\right)\right)-d .
$$

\section{Arithmetic lattice in $S L(n, \mathbb{R})$}

For a hyperbolic isometry $A \in S L(n, \mathbb{R})$, the translation length of $A$ satisfies

$$
\ell_{A} \geq \sqrt{2} \operatorname{arccosh} \frac{|\operatorname{tr} A|}{n} \geq \sqrt{2} \log \frac{|\operatorname{tr} A|}{n} .
$$

See [6] for a proof. Note here that the metric on $S L(n, \mathbb{R}) / S O(n)$ is normalized so that the natural totally geodesic embedding of $S L(2, \mathbb{R}) / S O(2)$ has the sectional curvature equal to -1 .

Let $L$ be a totally real quadratic extension of a totally real number field $F$ of degree $f$ over $\mathbb{Q}$ such that

$$
\begin{gathered}
\Gamma=S U\left(B=\left(b_{1}, \cdots, b_{m}\right), \tau ; \mathcal{O}_{D}\right)=\left\{g \in S L\left(m, \mathcal{O}_{D}\right) \mid\left(g^{\tau}\right)^{T} B g=B\right\} \\
\subset S L\left(n=m d, \mathcal{O}_{F}\right)
\end{gathered}
$$

is a uniform lattice, where $D$ is a central simple division algebra of degree $d$ over $L, b_{i} \in D^{\times}, \tau$ is an anti-involution of $D, \tau\left(b_{i}\right)=b_{i}$, and $\mathcal{O}_{D}$ is an order of $D$. Here $S U\left(B, \tau ; \mathcal{O}_{D}\right)^{\sigma}$ is compact for any nontrivial Galois embedding $\sigma$ of $F$. Note that for any $A \in \Gamma$, the eigenvalues of $A^{\sigma}$ have norm 1 , hence $\left|\operatorname{tr} A^{\sigma}\right| \leq n$.

Suppose $N(I)=\left|\mathcal{O}_{F} / I\right|$ is large for a prime ideal $I \subset \mathcal{O}_{F}$. Then for any $A=\left(a_{i j}\right) \in \Gamma(I)$ where

$$
\Gamma(I)=\Gamma \cap \operatorname{ker}\left(S L\left(n, \mathcal{O}_{F}\right) \rightarrow S L\left(n, \mathcal{O}_{F} / I\right)\right),
$$

$a_{i i}=1+c_{i}$ for $c_{i} \in I$ and $a_{i j} \in I$. Hence

$$
\left|\operatorname{tr} A^{\sigma}\right|=\left|\sum \sigma\left(1+c_{i}\right)\right|=\left|n+\sum \sigma\left(c_{i}\right)\right| \leq n .
$$

This implies that

$$
\left|\sum \sigma\left(c_{i}\right)\right| \leq 2 n
$$


Then

$N(I) \leq N\left(c_{1}+\cdots+c_{n}\right)=\left|c_{1}+\cdots+c_{n}\right| \Pi_{\sigma \neq i d}\left|\sigma\left(\sum c_{i}\right)\right| \leq(2 n)^{f-1}\left|c_{1}+\cdots+c_{n}\right|$.

Finally we get

$$
|\operatorname{tr} A|=\left|n+c_{1}+\cdots+c_{n}\right| \geq\left|c_{1}+\cdots+c_{n}\right|-n \geq \frac{N(I)}{(2 n)^{f-1}}-n .
$$

Hence we obtain for $A \in \Gamma(I)$

$$
\ell_{A} \geq \sqrt{2} \log \frac{|\operatorname{tr} A|}{n} \geq \sqrt{2} \log \left(\frac{N(I)}{n(2 n)^{f-1}}-1\right) \geq \sqrt{2} \log N(I)+c .
$$

Since $S L(n+1, \mathbb{R})$ is of type $A_{n}$, according to Table 1

$$
\left|S L\left(n+1, \mathcal{O}_{F} / I\right)\right| \leq N(I)^{n(n+2)} .
$$

Another way to construct an arithmetic lattice of $S L(n, \mathbb{R})$ is as follows. Let $D$ be a central division algebra of degree $d$ over $\mathbb{Q}$, such that $D$ splits over $\mathbb{R}$, and $\mathcal{O}_{D}$ an order. Then $\phi\left(S L\left(m, \mathcal{O}_{D}\right)\right)$ is an arithmetic subgroup of $S L(n=d m, \mathbb{R})$ for any embedding $\phi: D \rightarrow M(d, \mathbb{R})$. Then the same argument as above with $F=\mathbb{Q}$ goes through.

Since these two cases cover all types of arithmetic lattices in $S L(n+1, \mathbb{R})$, see [8], previous arguments give:

Theorem 2.1. The systol growth of arithmetic lattices in $S L(n+1, \mathbb{R})$ under the congruence cover is

$$
\operatorname{sys}_{1}\left(M_{I}\right) \geq \frac{\sqrt{2}}{n(n+2)} \log \left(\operatorname{vol}\left(M_{I}\right)\right)-d .
$$

Proof. It follows from $\operatorname{vol}\left(M_{I}\right)=\operatorname{vol}(M)\left[\Gamma: \Gamma_{I}\right]$ and $\left[\Gamma: \Gamma_{I}\right] \leq \mid S L(n+$ $\left.1, \mathcal{O}_{F} / I\right) \mid \leq N(I)^{n(n+2)}$.

Note that for $n=1, S L(2, \mathbb{R})$ is the isometry group of real hyperbolic plane, and the constant is $\frac{\sqrt{2}}{3}$, which is less than $\frac{4}{3}$. But Equation (2.1) is responsible for $\sqrt{2}$. Indeed in $S L(2, \mathbb{R})$ case, we have a better estimate

$$
\ell_{A} \geq 2 \log \frac{|\operatorname{tr} A|}{2}
$$

and hence the constant is $\frac{2}{3}$ instead of $\frac{\sqrt{2}}{3}$. To get the missing factor 2 , we need a better estimate for the length-trace inequality to get $N(I)^{2}$ factor.

This suggests that our constant is not optimal. On the other hand, the constant provided in [6] is cubic in $n$.

\section{UNIFORM LOWER BOUND FOR SYSTOLES OF NONUNIFORM ARITHMETIC MANIFOLDS IN COMPLEX HYPERBOLIC SPACE}

In this section we prove that 
Theorem 3.1. There exists a uniform lower bound for the length of closed geodesics in non-unform arithmetic lattices of $S U(n, 1)$ for any $n<N$, once $N$ is fixed.

First of all, we need a concept of complex Salem number.

Definition 3.2. A complex number $\lambda=r e^{i \theta}(r>1)$ is a complex Salem number if $\lambda$ is a root of monic polynomial over $\mathbb{Z}$ such that other roots except $\lambda, \bar{\lambda}$ and $\bar{\lambda}^{-1}, \lambda^{-1}$ all lie on the unit circle. The monic minimal polynomial of $\lambda$ over $\mathbb{Q}$ is called a Salem polynomial of $\lambda, s_{\lambda}(z)=s(z)$.

Here are some examples of minimal polynomials of complex Salem numbers.

$$
\begin{gathered}
1+x+x^{2}-x^{3}+x^{4}+x^{5}+x^{6} \\
1+x^{3}+x^{4}+x^{5}+x^{8} \\
1+x-x^{5}+x^{9}+x^{10}
\end{gathered}
$$

Theorem 3.3. Let $\Gamma \subset S U(n, 1)$ be a complex hyperbolic arithmetic lattice over $K$. Then for any hyperbolic isometry $\gamma$, the largest (in absolute value) eigenvalue is a complex Salem number.

Proof. Let $\gamma \in \Gamma$ be a hyperbolic isometry, hence

$$
\gamma^{m} \in S U(h, \mathcal{O}) \subset G L\left(N, \mathcal{O}_{K}\right)
$$

for some $m$. Indeed, one can take $N=2 n+2$. If $p(z)$ is the characteristic polynomial of $\gamma$ and $p^{\prime}(z)$ is the characteristic polynomial of $\gamma^{m}$, then $p^{\prime}(z)$ is defined over $\mathcal{O}_{K}$ since matrix in $G L\left(N, \mathcal{O}_{K}\right)$ has coefficients in $\mathcal{O}_{K}$. The roots of $p^{\prime}(z)$ are the $m$ th powers of the roots of $p(z)$. Since the coefficients of $p^{\prime}(z)$ are in $\mathcal{O}_{K}$, the roots of $p^{\prime}(z)$ are integral over $\mathcal{O}_{K}$. Since $\mathcal{O}_{K}$ is integral over $\mathbb{Z}$, the roots of $p^{\prime}(z)$ are integral over $\mathbb{Z}$, i.e. algebraic integers. Since the set of algebraic integers in $\mathbb{C}$ are integrally closed, the roots of $p(z)$ are algebraic integers. Hence its coefficients of $p(z)$ are in $\mathcal{O}_{K}$.

Furthermore $\gamma$ is conjugate to a matrix of the form (1.2). Hence $p(z)$ has a root $\lambda=r e^{i \theta}, r>1, \bar{\lambda}^{-1}$, and all other roots on the unit circle. Let $p(z)=$ $p_{1}(z) \cdots p_{k}(z)$ be a factorization into monic irreducible polynomials over $\mathcal{O}_{K}$ such that $p_{1}\left(\bar{\lambda}^{-1}\right)=0$. Then one can show that $\lambda, \bar{\lambda}^{-1}$ are roots of $p_{1}(z)$ as follows.

Suppose not. Then the constant term $w \bar{\lambda}^{-1}$ of $p_{1}(z)$ is in $\mathcal{O}_{K}$ where $w$ is some unit complex number. Choose non-identity $\sigma: K \rightarrow \mathbb{R}$ such that $\pm 1 \neq \sigma\left(w \bar{\lambda}^{-1}\right) \in \mathcal{O}_{\sigma K}$. Then the absolute value of $\sigma\left(w \bar{\lambda}^{-1}\right)$ cannot be 1 . But this contradicts that $\sigma\left(w \bar{\lambda}^{-1}\right)$ is a root of $p^{\sigma}(z)$ which is a characteristic polynomial of $\gamma^{\sigma}$ in a compact unitary group $S U\left(h^{\tau_{j}}, A \otimes_{l} \mathbb{C}\right)$ for some $\tau_{j}: l \rightarrow \mathbb{C}$.

Suppose $\tau_{i}: l \rightarrow \mathbb{C}, i=1, \cdots, d=[l: \mathbb{Q}]$ are embeddings of $l$ into $\mathbb{C}$ and $\tau_{1}=$ $i d$ and $\tau_{2}=\bar{\tau}_{1}$ is a complex conjugation, $\tau_{2 k}=\bar{\tau}_{2 k-1}$. Then $\tau_{2 k-1} \mid K: K \rightarrow \mathbb{R}$ are Galois embeddings of $K$ into $\mathbb{R}$. Note that $p_{1}(z) p_{1}^{\tau_{2}}(z)$ has $\lambda, \bar{\lambda}, \lambda^{-1}, \bar{\lambda}^{-1}$ 
as roots. Set $p_{1}^{*}(z)=\Pi p_{1}^{\tau_{i}}(z)$. Since $p_{1}^{*}$ is invariant under $\tau_{i}, p_{1}^{*}(z)$ is over $\mathbb{Z}$. Since $p_{1}(z)$ is a factor of $p(z)$, each $p_{1}^{\tau_{i}}$ is a factor of $p^{\tau_{i}}(z)$ for $i>1$. But since $h^{\tau_{i}}$ is unitary for $i=2 k-1>1, p^{\tau_{i}}(z)$ must have roots all on the unit circle. This shows that $p_{1}^{*}(z)$ has roots all on the unit circle except $\lambda, \lambda^{-1}, \bar{\lambda}, \bar{\lambda}^{-1}$. If $s(z)$ is an irreducible minimal polynomial over $\mathbb{Z}$ of $\lambda, s(z)$ must divide $p_{1}^{*}(z)$. If $s(z)=p_{1}^{*}(z)$, then $\lambda$ is a complex Salem number and $s(z)$ is a Salem polynomial. Hence it suffices to show that $p_{1}^{*}(z)$ is irreducible over $\mathbb{Z}$. Suppose $p_{1}^{*}(z)=s(z) h(z)$ for some monic polynomial $h(z)$ over $\mathbb{Z}$. For a root $r$ of $h(z)$, it is a root of $p_{1}^{\tau}(z)$ for some $\tau=\tau_{j}$. Then $p_{1}^{\tau}(z)$ is a minimal polynomial of $r$ over $\tau(K)$ since the same thing is true for $p_{1}(z)$ and any root of it. Hence $p_{1}^{\tau}$ divides $h$ in $\tau(K)[z]$. Applying $\tau^{-1}$, since $h^{\tau^{-1}}=h$ ( $h$ is over $\mathbb{Z})$, we see that $p_{1}$ divides $h$ in $K[z]$. Hence $\lambda$ is a root of $h(z)$, but $\lambda$ is a simple root of $p_{1}^{*}(z)$. Hence $s(z)=p_{1}^{*}(z)$.

Note that the degree of $\lambda$ over $\mathbb{Q}$ is $\leq 2(n+1)[l: \mathbb{Q}]$. If $\Gamma$ is non-uniform, then necessarily $K=\mathbb{Q}$.

Since there are only finitely many monic polynomials of bounded degree with bounded Mahler measure [4], the set of complex Salem numbers of bounded degree over $\mathbb{Q}$ has a least element. Consequently we have

Corollary 3.4. If $\Gamma \subset S U(n, 1)$ is a non-uniform arithmetic lattice, then the length of a closed geodesic is uniformly bounded below for any $n \leq N$ for a fixed $N$.

For real hyperbolic case, the above corollary is proved in [3].

\section{REFERENCES}

[1] P. Buser and P. Sarnak, On the period matrix of a Riemannian surface of large genus (with an appendix by J. Conway and N. Sloan), Inventiones Math. 117 (1994), 27-56.

[2] V. Emery, I. Kim and P. Murillo, Lower bound for systole of congruence coverings of quaternionic hyperbolic manifolds, preprint.

[3] V. Emery, J. Ratcliffe and S. Tschantz, Salem numbers and arithmetic hyperbolic groups, Trans. of AMS, 372 (1) (2019), 329-355.

[4] E. Ghate and E. Hironaka, The arithmetic and geometry of Salem numbers, Bull. Amer. Math. Soc. 38 (2001), 293-314.

[5] M. Gromov, Systoles and intersystolic inequalities, IHES Séminaires \& Congrés 1, 1996.

[6] S. Lapan, B. Linowitz, and J. Meyer, Systole inequalities up congruence towers for arithmetic locally symmetric spaces, preprint.

[7] D. McReynolds, Arithmetic lattices in $S U(n, 1), \mathrm{PhD}$ Thesis.

[8] D. Morris, Introduction to Arithmetic groups, Deductive Press, 2015. xii+475 pp.

[9] P. Murillo, On arithmetic manifolds with large systole. PhD Thesis. IMPA 2017.

Inkang Kim: School of Mathematics, KiAS, Heogiro 85, Dongdaemun-gu Seoul, 02455, Republic of KoreA

E-mail address: inkang@kias.re.kr 